\title{
The Usage of Emoji in Tourism-Related Instagram Posts: Suggestions from a Marketing Perspective
}

\author{
Viktoria Distel, Roman Egger ${ }^{(\bowtie)}$ (D), Ugljesa Petrovic, \\ Viet Linh Phan, and Simon Wiesinger \\ Salzburg University of Applied Sciences, Salzburg, Austria \\ \{vdistel. imte-m2019, roman. egger, \\ upetrovic. imte-m2019, vphan. imte-m2019, \\ swiesinger.imte-m2019\}@fh-salzburg.ac.at
}

\begin{abstract}
The relevance of emoji in social media marketing has attracted tremendous interest from academics and marketing professionals alike ever since emoji became a fixed component in user-to-user and business-to-user communication on online platforms such as Instagram and Facebook. Using a quantitative research approach in the form of a self-administered online survey in an experimental setting, the purpose of this study is to investigate what type of emoji positively impacts consumer behaviour, purchase intention, and user interaction in tourism-related Instagram posts. This research is novel in that it bridges the usage of emoji in the context of social media and tourism. The findings support tourism managers in the practical use of emoji for social media marketing campaigns on Instagram and show that (positive) face emoji evoke more positive emotions than non-face emoji.
\end{abstract}

Keywords: Emoji $\cdot$ Instagram $\cdot$ Social media advertising $\cdot$ Tourism marketing

\section{Introduction}

With the rise of digital communication, social media platforms such as Facebook, Twitter, and Instagram have transformed into powerful communicational mediums as they allow users to express feelings, share everyday life moments, and connect with others [1]. In particular, Instagram, initially launched as a photo-sharing platform, has become one of the world's most popular channels, with over one billion active users. Beyond its visual-centred nature, Instagram acts as a network for several industries such as services, marketing, and tourism to grow their business. Increasingly, the potentials of Instagram have also been recognised by tourism service providers and destination marketing organisations (DMOs) for the creation of effective marketing strategies [2].

In addition to using videos and images for marketing purposes, emojis offer brands the opportunity to connect with the target market [3] on Instagram using an emotional touch. Essentially, emojis are facial expressions that allow users to express their feelings, moods, or emotions. As graphic symbols that show vehicles, food and 
beverages, weather, flags, animals, and so on [4], two main types of emoji can be identified; namely, face emoji (expressing human emotion and feelings, e.g., 2 ) and non-face emoji (which do not involve human faces, e.g., $\bigcirc \square$ ) [5]. Emoji can emphasise written text language as they are the most evolved form of available punctuation [6].

Seeing that emojis and emoticons are highly pervasive in our daily lives [7], recent studies have focused on customer purchase intentions relating to the appearance of emoji in advertising [8, 9]. For instance, scholars suggested that experimental purchases such as tourism products and services can stimulate emotions that positively increase emotional behaviour [10]. That is, travel-related products tend to influence emotions, whereby emoji can play a crucial role since they express an emotional mood as well. However, while emoji usage in tourism enterprise communications has been highlighted [11], the linkage between emoji and their significance in tourism advertising remains fragmented.

Meanwhile, although earlier literature has discussed Instagram advertising of DMOs [1, 12] and highlighted the vital role of user-generated content (UGC) on destination image formation [2,13], the relation of user-generated content and the usage of emoji in tourism-related posts on Instagram is still an underexplored area. Likewise, while some scholars have looked into emoji and their meaning in regard to customer attention [14, 15], there are limited studies that focus on emoji and their impacts on the efficiency of tourism-related advertisements, especially on Instagram [16].

Owing to the rising interest in emoji around various research streams [16], this study aims to contribute to tourism research by examining what type of emoji attracts the most attention on tourism-related Instagram posts as well as what type of emoji creates more positive emotions in such advertisements. Additionally, this study assesses the type of emoji that creates the most user interaction. The specific research questions are as follows:

(1) How does the usage of specific types of emoji (face emoji and non-face emoji) in tourism-related Instagram posts influence an individual's attention to the post?

(2) What type of emoji help to raise positive emotions in tourism-related Instagram posts?

(3) How can emoji be useful for tourism managers in managing tourism marketing activities?

(4) What type of emoji help to create more interactions with the post?

From a practical point of view, this study's findings can support destination management and tourism marketers in optimising their social media advertising and effective involvement of emoji in Instagram posts. 


\section{Literature Review and Hypothesis Development}

\subsection{Emoji and Social Media}

As part of computer-mediated communication, emoji are widely used, and some emoji have nearly achieved universal understanding [17]. Emoji have been used in different ways and contexts on social media platforms including Instagram, Facebook, Twitter, and WhatsApp. They allow people with different cultural backgrounds to communicate more effectively and initiate interactions when necessary. It is believed that emoji can solve potential misunderstandings experienced in intercultural communication [18]. According to theory in social semiotics, emoji serves as a means of promoting shared values and affiliation between users [11]. This concept is expressed via a semiotic that establishes interpersonal meaning within the attitude system and a semiotic establishing experiential meaning.

When emoji were used, readers found a higher level of positive emotion than when no emoji were presented [17]. For non-face emoji, even though they do not show facial expressions, they still bring positive meanings to a message. Furthermore, emoji help to convey the meaning of a message more efficiently [19]; they can significantly reduce the ambiguity of a sentence and are able to change one's interpretation in terms of affection [5]. Hence, emoji can help to catch the attention of readers in a shorter period of time [15] and help to create a digital message with nuances and depth, resembling a face-to-face conversation more closely [14]. Other scholars suggested that a message is also considered to be more personalised when that message includes emoji [15]. They also point to the elaboration likelihood model, which explains the influence of emoji on the reader's attention. The model implies that information may persuade people with positive cues when they can be processed without much thinking, and personalisation can positively affect information processing [20].

Facial emoji encourage users to express different emotions that may not be as easily portrayed in online communication. People select suitable emoji that fits their current emotion/mood from a predefined list of emotional expressions. While, during the process of exchanging information on social network sites, this may seem like an extraordinary action, this is in fact an exercise that fosters our emotional honesty because, often times, individuals may be unable to express their true feelings due to social barriers [14]. Moreover, notably, visual images deliver higher emotional effects than just words [19]. Using emoji in a non-judgmental setting thus helps to understand our emotional state and to communicate it more openly [14]. However, this depends on the specific context and the individual perception of the respective emoji. Regardless, emoji convey a particular emotion and reinforce a message [21], which support us in conveying our socially challenging emotions and, by that, in acknowledging and accepting that emotion as a part of ourselves [12]. Accordingly, the following hypothesis has been formulated:

H1: The appearance of positive face emoji in tourism related Instagram posts raises more positive emotions than non-face emoji do. 


\subsection{Emoji, Consumer Behaviour, and Purchase Intention}

While many functions and facets of emoji in communication have been explored in social media research, the usage of emoji and their effect on consumer behaviour and purchase intention is another essential component that needs to be considered when aiming to gain insights on the precise usage of emoji. To date, emoji are widely prevalent in marketing campaigns [9]; in other words, companies pursue new ways of communication that may positively influence the emotions of potential consumers. Hence, emoji are well recognised as a tool for social media advertising [3]. Recent literature suggest that emoji in promotional materials encourage users to respond to the message [23] and, regarding hedonic products, increase the effect of purchase intention [8]. Other scholars report an increasing number of emoji in marketing campaigns and point out that emoji in advertisements can positively impact targeted consumers because users appreciate the service more and the level of their satisfaction increases [9].

Notably, stimulation of consumers' emotional behaviour is particularly relevant when it comes to experience-orientated products, such as tourism services [10]. This study assumes the purchase intention of potential consumers to be equivalent to travel intention since an advertisement from an official destination management organisation on Instagram aims to bring travellers to the promoted destination. This has led to the following hypothesis:

H2: The appearance of emoji in tourism-related Instagram advertisements influences users' travel intention.

\subsection{Instagram and Emoji in Tourism}

Instagram has become a popular platform that triggers creativeness and self-promotion of tourism destinations $[12,22]$. When applied strategically, user-generated content (UGC) can be highly beneficial for tourism organisations [24] because it enhances the destination image [12, 24]. Seeing that travellers can be viewed as low-cost marketers and brand ambassadors, research has found that pictures taken by users and reposted by DMOs improve engagement on Instagram [25]. Other scholars emphasise UGC as a crucial factor for destination image because user-generated Instagram pictures tend to present more diverse attractions than traditional brochures and travel guides [26]. As such, more tourism operators use Instagram to promote destinations and consider Instagram to be an online travel album where all tourists have access [12].

In terms of emoji usage in the tourism industry, a transformation in company communications including emoji on social media platforms has appeared [27]. As a result, travel organisations use emoji when communicating with tourists, leading to an increased human touch in business communications. Tourism is an industry that should strongly rely on the usage of emoji when considering tourism enterprises and their social media strategies as the integration of emoji brings more fun and friendly interactions to the customer base, which eventually leads to better business development [28]. In particular, it has been recognised that non-face emoji enhance reader trust by reducing message ambiguity [5]. As such, this study tests for the types of emoji that help to create effective UGC with the following hypothesis: 
H3: The appearance of non-face emoji in tourism related Instagram posts helps to promote more engagement (likes, shares, and comments) than face emoji posts do.

\section{Methodology}

This research adopted a quantitative method approach with an online questionnaire in an experimental setting. More specifically, a within-subjects research design was applied (see Fig. 1) in which participants were asked to randomly evaluate a set of two pictures with a caption with text and a face emoji, two pictures with a caption with text and a non-face emoji, and two pictures with a caption with text only. The images were selected in a way that they do not show landmarks or people and mix indoor and outdoor shots.

The emoji were selected based on "The Most Frequent Emoji" list in 2019 [29],

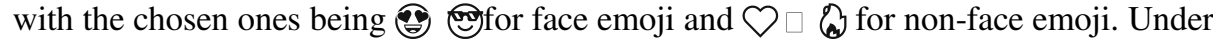
each Instagram post, a list of questions were asked to assess participants' attitudes, travel intention, and feeling (e.g., joy, interest, and contentment) towards the post. They were also asked to rate their perception of the picture and reactions to the post (i.e., like, share, and comment) based on a 6-point Likert scale to avoid the central tendency bias.

Participants were recruited via email and personal Instagram accounts using a nonprobability and purposive sampling technique. The inclusion criterion were adult participants over 18 years old as they are legally entitled to purchase tourism products and services on their own. To ensure that participants were familiar with the nature of Instagram, individuals without an active Instagram account were not considered In this way, lurkers were excluded. Altogether, the sample ultimately consisted of 182 respondents.
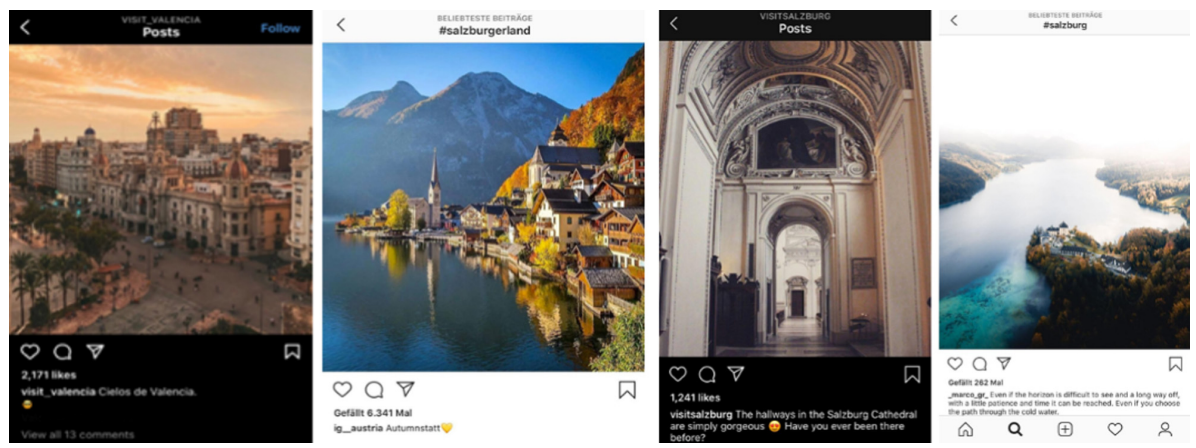

Fig. 1. Example of Instagram posts used in the survey. 


\section{Results}

\subsection{Demographic Profile of Respondents}

Overall, as presented in Table 1, the majority of participants were female (75.3\%) and skewed toward the younger population, aged between 18 and 29 (89\%). In addition, $52.2 \%$ of participants agreed that they use Instagram to upload pictures and stories, while $47.8 \%$ only check the feed. The majority (61.5\%) used Instagram several times a day.

Table 1. Demographic information $(n=182)$.

\begin{tabular}{l|l|l|l}
\hline \multicolumn{2}{l|}{ Gender } & \multicolumn{3}{l}{ Activeness } \\
\hline $\begin{array}{l}\text { Male } \\
\text { Female }\end{array}$ & $24.7 \%$ & $\begin{array}{l}\text { To upload pictures and stories } \\
\text { To check the feed }\end{array}$ & $52.2 \%$ \\
\hline Age & & Frequency & \\
\hline $18-23$ & $46.7 \%$ & Multiple times per hour & $16.5 \%$ \\
$24-29$ & $42.3 \%$ & Several times per day & $61.5 \%$ \\
$30-35$ & $8.8 \%$ & One to two times per day & $13.2 \%$ \\
36 or above & $2.2 \%$ & $\begin{array}{l}\text { Three to seven times per week } \\
\text { One to two times per week }\end{array}$ & $3.3 \%$ \\
& & One & \\
\hline
\end{tabular}

\subsection{The Influence of Emoji on Emotion, Travel Intention, and Engagement}

A non-parametric ANOVA Friedman test was chosen to determine the difference between participants' emotion when seeing Instagram posts with face emoji, non-face emoji, and no emoji at all. The result suggested a statistically significant difference between the levels of emotion. The Dunn-Bonferroni post hoc test (Table 2) showed that, even when comparing in pairs, the levels of emotion towards the three types of posts were all significantly different. To be more specific, the post with positive face emoji created the most positive emotions, while the post with non-face emoji provoked the least positive emotion. Hence, H1 can be accepted.

Likewise, the results indicated a statistically significant difference between the travel intention generated from the three types of posts (Table 2). Looking at the DunnBonferroni test outcome, travel intention towards the posts with face emoji and no emoji was found to be statistically significant. Yet, there was no statistically significant difference when comparing travel intention towards the posts with non-face emoji and no emoji. Moreover, based on the mean ranks, the post with face emoji showed the highest mean value regarding travel intention. Thus, $\mathrm{H} 2$ is only partially supported since face emoji positively influenced travel intention while the appearance of non-face emoji showed no statistical difference regarding travel intention compared to Instagram posts with no emoji involved.

Concerning Instagram users' engagement, the Friedman test revealed a statistically significant difference for "like", "comment", and "share" when users saw posts with face emoji, non-face emoji, and no emoji at all. Turning to the results of the post-hoc 
test, a statistically significant difference could be found between the intention to "like" a post with face-emoji and with non-face emoji; people were most likely to like a post with face emoji, followed by no emoji and non-face emoji in the caption. However, the post hoc test presented no significant difference when comparing participants' commenting and sharing behaviour in pairs. The results indicated that the presence of face emoji and non-face emoji neither encouraged users to comment nor to share the post when compared to no emoji at all. Thus, hypothesis 3 must be rejected. Yet, based on the mean rank, it showed that people do indeed tend to comment and share posts with face-emoji the most, followed by posts with non-face emoji and no emoji at all.

Table 2. Result of Friedman and Dunn-Bonferroni tests.

\begin{tabular}{|c|c|c|c|c|c|}
\hline \multirow[t]{2}{*}{ Constructs } & \multicolumn{3}{|c|}{ Friedman tests } & \multicolumn{2}{|l|}{ Dunn-Bonferroni test } \\
\hline & Chi-square & Asymp. Sig. & Mean rank & Pairs & Sig. \\
\hline \multirow{2}{*}{$\begin{array}{l}\text { Emotion } \\
\text { Face emoji } \\
\text { Non-face emoji } \\
\text { No emoji }\end{array}$} & \multirow[t]{2}{*}{63} & \multirow[t]{2}{*}{.000} & & & \\
\hline & & & $\begin{array}{l}1.58 \\
2.38 \\
2.04\end{array}$ & $\begin{array}{l}\text { Face emoji \& Non-face emoji } \\
\text { Face emoji \& No emoji } \\
\text { No emoji \& Non-face emoji }\end{array}$ & $\begin{array}{r}.000 \\
.000 \\
.005\end{array}$ \\
\hline \multirow{2}{*}{$\begin{array}{l}\text { Travel intention } \\
\text { Face emoji } \\
\text { Non-face emoji } \\
\text { No emoji }\end{array}$} & \multirow[t]{2}{*}{34.8} & \multirow[t]{2}{*}{.000} & & & \\
\hline & & & $\begin{array}{l}1.67 \\
2.20 \\
2.13\end{array}$ & $\begin{array}{l}\text { Face emoji \& Non-face emoji } \\
\text { Face emoji \& No emoji } \\
\text { No emoji \& Non-face emoji }\end{array}$ & $\begin{array}{l}.000 \\
.000 \\
1.000\end{array}$ \\
\hline \multirow{2}{*}{$\begin{array}{l}\text { Like } \\
\text { Face emoji } \\
\text { Non-face emoji } \\
\text { No emoji }\end{array}$} & \multirow[t]{2}{*}{41.6} & \multirow[t]{2}{*}{.000} & & & \\
\hline & & & $\begin{array}{l}1.66 \\
2.25 \\
2.09\end{array}$ & $\begin{array}{l}\text { Face emoji \& Non-face emoji } \\
\text { Face emoji \& No emoji } \\
\text { No emoji \& Non-face emoji }\end{array}$ & $\begin{array}{l}.000 \\
.000 \\
.406\end{array}$ \\
\hline \multirow{2}{*}{$\begin{array}{l}\text { Comment } \\
\text { Face emoji } \\
\text { Non-face emoji } \\
\text { No emoji }\end{array}$} & \multirow[t]{2}{*}{11.9} & \multirow[t]{2}{*}{.000} & & & \\
\hline & & & $\begin{array}{l}1.86 \\
2.04 \\
2.10\end{array}$ & $\begin{array}{l}\text { Face emoji \& Non-face emoji } \\
\text { Face emoji \& No emoji } \\
\text { No emoji \& Non-face emoji }\end{array}$ & $\begin{array}{l}.237 \\
.073 \\
1.000\end{array}$ \\
\hline \multirow{2}{*}{$\begin{array}{l}\text { Share } \\
\text { Face emoji } \\
\text { Non-face emoji } \\
\text { No emoji }\end{array}$} & \multirow[t]{2}{*}{11.4} & \multirow[t]{2}{*}{0.003} & & & \\
\hline & & & $\begin{array}{l}1.85 \\
2.07 \\
2.08\end{array}$ & $\begin{array}{l}\text { Face emoji \& Non-face emoji } \\
\text { Face emoji \& No emoji } \\
\text { No emoji \& Non-face emoji }\end{array}$ & $\begin{array}{l}.101 \\
.083 \\
1.000\end{array}$ \\
\hline
\end{tabular}

\subsection{Factors Influencing Travel Intention}

Finally, in addition to the effect of emoji, Table 3 presents other external factors in a post that may influence one's travel intention. On the whole, the findings concluded that bright colours and saturated chroma ("the picture is beautiful") were the most compelling factor, followed by the caption of a post ("the caption of the post is interesting"). Similarly, when looking at posts with face emoji, brightness and chroma as well as the caption were also found to be influential factors. 
Table 3. Instagram post factors influencing travel intention.

\begin{tabular}{l|l|l|l|l}
\hline & $\begin{array}{l}\text { Number of likes } \\
\text { and comments }\end{array}$ & $\begin{array}{l}\text { Brightness \& } \\
\text { chroma }\end{array}$ & $\begin{array}{l}\text { Trust towards } \\
\text { account }\end{array}$ & $\begin{array}{l}\text { Caption of the } \\
\text { post }\end{array}$ \\
\hline $\begin{array}{l}\text { Selected } \\
\text { (times) }\end{array}$ & 21 & 496 & 95 & 210 \\
\hline Face emoji & & 198 & & 48 \\
\hline \multirow{2}{*}{$\begin{array}{l}\text { Non-face } \\
\text { emoji }\end{array}$} & & 130 & & 42 \\
\hline No emoji & & 165 & & 43 \\
\hline
\end{tabular}

\section{Discussion}

Essentially, emoji add a positive meaning to the message (caption) [5], which, in turn, positively influences one's perception and instills a positive impression [30]. This study supports the appearance of (positive) face emoji in tourism-related Instagram posts promoting more positive emotions than non-face emoji. However, the result regarding the levels of emotions between posts with non-face emoji and without any emoji is not coherent with earlier literature claiming that non-face emoji also adds positive emotion to a post [5].

Meanwhile, face emoji in an Instagram post was also found to trigger one's intention to travel to a destination. This finding is supported by recent literature, suggesting that the presence of emoji on social media contributes to business development since it brings about a more favourable impression for the brand [11]. Subsequently, an increase in customers' willingness to purchase due to the inclusion of emoji echoes the context of buying hedonic products [8]. Thus, it can be concluded that the influence of positive face emoji on purchase intention also applies to destination marketing on Instagram. Face emoji create positive emotions for Instagram users, leading to an increase of their desire to travel; however, it is worth mentioning that not all emoji have the same effect. For instance, using non-face emoji in tourism-related Instagram posts generates a non-significant or less positive result than when no emoji is used. With regards to user engagement, users' liking, commenting, and sharing behaviours on Instagram have been recognised as crucial factors in promoting a destination [2]. In the same vein, this study's findings disclose that posts with positive face emoji attract the most "likes". This is consistent with the aforementioned results in which using positive emoji tends to generate the most positive emotion (e.g., joy, interest, and contentment), thereby obtaining the highest number of "likes".

On the other hand, since commenting requires more effort than clicking the "like" button, the more comments a post receives responds to a more profound level of user engagement [2]. When it comes to sharing, it also takes more time to consider sharing a post from DMOs since it reveals one's interest(s) to their friends, family, and other account followers [31]. This content can, in turn, influence other people's decision about a particular destination at a later stage [32]. Thus, the lack of significant difference regarding the likelihood that participants would comment or share the three 
types of posts shows that it is challenging to encourage Instagram users to comment or share Instagram posts posted by a DMO, despite the presence of emoji in the caption.

While the positive influence of positive face emoji on travel intention has already been examined, it is interesting to further investigate whether the desire to visit a destination is based solely on the effect of face emoji. First, in line with the literature, bright pictures with numerous colours activate a variety of senses, leading to higher popularity of a post [33]. That is, the findings suggest that the quality, colours, and brightness of pictures are also influential when it comes to travel intention. This result can also be explained through another recent study [34], stating that the brightness and colours of a picture affect viewers' emotion. Nevertheless, different from previous literature, where a post with a high number of likes and shares on Facebook can lead to higher purchase intention [35], our findings imply that the same notion may not be applicable to Instagram in the context of destination marketing. Regardless, all in all, the results support a compounding positive effect when face emoji is embedded in a caption along with "beautiful pictures" consisting of bright colours and chroma.

\section{Conclusion}

Until now, research on Instagram emoji has been limited and primarily focused on how emoji inserted in captions can influence post popularity [36]. Other researchers have mainly explored the impact of emoji in the context of promoting hedonic products [8, $9,28]$. This research thus adds knowledge to the existing body of literature related to semiotics and tourism. By connecting the effect of emoji to the domain of tourism marketing, this research reinforces that emoji may not only prompt a more positive impression towards a brand but also towards a place. Moreover, evidence revealed that, in the case of tourism marketing on Instagram, non-face emoji in captions may not bring about the same desired effect in generating positive marketing outcomes. This sheds light on a different perspective compared to existing research on how non-face emoji can also bring positive emotion when used in instant messaging [5]. In essence, this study contributes to the emerging field of marketing communications and semiotics in tourism research by identifying whether specific types of emoji (face emoji and nonface emoji) in tourism-related Instagram posts influence an individual's attention and behaviour.

The findings also offer numerous implications for destination marketers concerning emoji usage in digital marketing communications. Throughout this study, posts with positive emoji have performed better across all categories. Thus, marketers are advised to include emoji in their rhetoric strategy for social media marketing as it facilitates effective communication with potential travelers. Especially the usage of positive face emoji on Instagram can be beneficial for increasing positive impressions and feelings towards the promoted destination. However, destination marketers should also consider that different emoji serve different purposes. Since the findings show that the positive effect created by face emoji is associated with a higher intention of travel behaviour, it is advisable that for promotional posts aimed at increasing travel intention, preference should be given to positive face emoji over non-face emoji in order to guarantee more interactive and appealing content for DMOs and marketers. Furthermore, a picture's 
quality, including brightness and chroma, is another essential factor that marketers should pay attention to [2]. To conclude, in order to make the best out of an Instagram post, it is advised to include positive face emoji in the caption and high-quality pictures with bright colours.

Nonetheless, there are several limitations to this study that should be highlighted. First, the sample of the participants was unbalanced, with the majority being females. The sampling technique also resulted in an age skew. Even though a selection of images was utilized for this study, the biggest limitation lies in the fact that it is hard to control other stimuli and post elements. Moreover, considering the number of social media platforms available to date, the findings of this study are limited due to the focus placed on Instagram users.

For future research, scholars are advised to look at other types of hedonic advertising products. It is also recommended to investigate how different settings, advertisements, placements, and number of emoji influence tourists' online behaviour. Similarly, exploring which type of information source is more powerful in eliciting travel intention could be of interest for future research. Furthermore, scholars could study whether emojis can influence interest in information intensification. Future research could also zoom in on other social media platforms to investigate whether the same effects hold true regarding consumer behaviour and advertisement perceptions. Another interesting perspective for future research would be the comparison of emoji usage across different social media platforms. Finally, adding more face emoji and nonface emoji would additionally be a valuable direction to offer deeper insights.

\section{References}

1. Yu C-E, Sun R (2019) The role of Instagram in the UNESCO's creative city of gastronomy: a case study of Macau. Tour Manage 75:257-268

2. Yu C-E, Xie SY, Wen J (2020) Coloring the destination: the role of color psychology on Instagram. Tourism Manage 80:104110

3. Ge J, Gretzel U (2018) Emoji rhetoric: a social media influencer perspective. J Mark Manag 34:1272-1295

4. Novak PK, Smailović J, Sluban B (2015) Mozeti, č I. Sentiment of emoji 10:e0144296

5. Riordan MA (2017) The communicative role of non-face emojis: affect and disambiguation. Comput Hum Behav 76:75-86

6. Favilla EJ (2017) A world without whom: the essential guide to language in the buzzfeed age. Bloomsbury Publishing, USA

7. Prada M, Rodrigues DL, Garrido MV et al (2018) Motives, frequency and attitudes toward emoji and emoticon use. Telematics Inform 35:1925-1934

8. Das G, Wiener HJ, Kareklas I (2019) To emoji or not to emoji? Examining the influence of emoji on consumer reactions to advertising. J Bus Res 96:147-156

9. Hsu L, Chen Y-J (2020) Neuromarketing, subliminal advertising, and hotel selection: an EEG study. Australas Mark J 28:200-208

10. Su L, Cheng J, Swanson SR (2020) The impact of tourism activity type on emotion and storytelling: the moderating roles of travel companion presence and relative ability. Tourism Manage 81:104138 
11. Bezirgan M, Kaya İ (2019) Digital communications and emoji use in tourism enterprises. Theory Pract Soc Sci 10

12. Fatanti MN, Suyadnya IW (2015) Beyond user gaze: how instagram creates tourism destination brand? Proc Soc Behav Sci 211:1089-1095

13. Arefieva V, Egger R, Yu J (2021) A machine learning approach to cluster destination image on Instagram. Tourism Manage 85:104318

14. Elder AM (2018) What words can't say. JICES 16:2-15

15. Willoughby JF, Liu S (2018) Do pictures help tell the story? An experimental test of narrative and emojis in a health text message intervention. Comput Hum Behav 79:75-82

16. Bai Q, Dan Q, Mu Z et al (2019) A systematic review of emoji: current research and future perspectives. Front Psychol 10:2221

17. Riordan MA (2017) Emojis as tools for emotion work: communicating affect in text messages. J Lang Soc Psychol 36:549-567

18. Onursoy S (2018) Using emojis: self-presentation and different meaning creation approaches. Galatasaray Üniversitesi İleti-ş-im Dergisi

19. Huang AH, Yen DC, Zhang X (2008) Exploring the potential effects of emoticons. Inf Manage 45:466-473

20. Petty R, Cacioppo J (1986) The elaboration likelihood model of persuasion'. Adv Exp Soc Psychol 19:123-205

21. Derks D, Fischer AH, Bos AE (2008) The role of emotion in computer-mediated communication: a review. Comput Hum Behav 24:766-785

22. Daxböck J, et al (2021) The implicit and explicit motivations of tourist behaviour in sharing travel photographs on Instagram: a path and cluster analysis. In Information and Communication Technologies in Tourism 2021 pp 244-255. Springer, Cham. https://doi. org/10.1007/978-3-030-65785-7_22

23. Vaiciukynaite E, Massara F, Gatautis R (2017) An investigation on consumer sociability behaviour on facebook. EE 28

24. Hanan H, Putit N (2013) Express marketing of tourism destinations using Instagram in social media networking. Hospital Tourism 471-474

25. Iglesias-Sánchez PP, Correia MB, Jambrino-Maldonado C et al (2020) Instagram as a cocreation space for tourist destination image-building: algarve and costa del sol case studies. Sustainability 12:2793

26. Paül i Agustí D (2018) Characterizing the location of tourist images in cities. differences in user-generated images (Instagram), official tourist brochures and travel guides. Ann Tour Res 73:103-115

27. Dijkmans C, Kerkhof P, Beukeboom C (2020) Adapting to an emerging social media landscape: the rise of informalization of company communication in tourism. In: Neidhardt $\mathrm{J}$, Wörndl W (eds) Information and Communication Technologies in Tourism 2020. Springer, Cham, pp 3-14. https://doi.org/10.1007/978-3-030-36737-4_1

28. Krystev V, Efe R, Atasoy E (2019) Theory and Practice in Social Sciences. Recep Efe

29. Unicode (2019) Emoji frequency. https://home.unicode.org/emoji/emoji-frequency/. Accessed 01 Jun 2020

30. Coyle MA, Carmichael CL (2019) Perceived responsiveness in text messaging: the role of emoji use. Comput Hum Behav 99:181-189

31. Buhalis D, Foerste M (2015) SoCoMo marketing for travel and tourism: empowering cocreation of value. J Destin Mark Manag 4:151-161

32. Zeng B, Gerritsen R (2014) What do we know about social media in tourism? A review. Tourism Management Perspectives 10:27-36

33. Sabate F, Berbegal-Mirabent J, Cañabate A et al (2014) Factors influencing popularity of branded content in Facebook fan pages. Eur Manag J 32:1001-1011 
34. Gao X-P, Xin JH, Sato T et al (2007) Analysis of cross-cultural color emotion. Color Res Appl 32:223-229

35. Dehghani M, Tumer M (2015) A research on effectiveness of Facebook advertising on enhancing purchase intention of consumers. Comput Hum Behav 49:597-600

36. Carta S, Podda AS, Recupero DR et al (2020) Popularity prediction of Instagram posts. Information 11:453

Open Access This chapter is licensed under the terms of the Creative Commons Attribution 4.0 International License (http://creativecommons.org/licenses/by/4.0/), which permits use, sharing, adaptation, distribution and reproduction in any medium or format, as long as you give appropriate credit to the original author(s) and the source, provide a link to the Creative Commons license and indicate if changes were made.

The images or other third party material in this chapter are included in the chapter's Creative Commons license, unless indicated otherwise in a credit line to the material. If material is not included in the chapter's Creative Commons license and your intended use is not permitted by statutory regulation or exceeds the permitted use, you will need to obtain permission directly from the copyright holder.

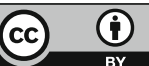

